\title{
TRATAMIENTO DE LA FASE INICIAL DEL SHOCK HEMORRAGICO EN GINECO OBSTETRICIA CON GELATINA POLIMERIZADA*
}

\author{
Dr. Alberto Duarte Contreras** \\ Dr. Samuel Jiménez A.***
}

El shock hemorrágico representa en la práctica gineco-obstétrica una emergencia bastante frecuente que ensombrece seriamente, en muchos casos, el pronóstico inmediato y aún tardío de las pacientes.

Tenemos la impresión de que en el momento actual en nuestro medio, encontramos un aumento significativo del shock hemorrágico en pacientes jóvenes, debido al mayor e inusitado número de abortos provocados.

La frecuencia del shock hemorrágico en pacientes gineco obstétricas la podemos investigar con relativa facilidad si recordamos que en la primera mitad del embarazo la vamos a encontrar en abortos, en embarazos ectópicos y en embarazos molares; en la segunda mitad se nos presenta en placenta previa, en desprendimiento prematuro de placenta normoincerta y en ruptura uterina; y durante el parto en lesiones cervicales o del canal genital, en cesáreas, en atonía uterina, en retención de placenta y restos, en eversión uterina y por defectos de mecanismos de coagulación. En ginecología es un poco más difícil, de ordinario, esta valoración, pues a más de presentarse en pacientes de mayor edad por lesiones casi siempre malignas, se encuentra con

alguna frecuencia durante el acto quirúrgico y aún en el postoperatorio inmediato $(1,2)$.

Algunos autores sostienen que en pacientes jóvenes se necesita una pérdida del 30 al $50 \%$ del volumen sanguíneo para que se instaure el shock (3). Afortunadamente este grupo de pacientes es el que encontramos de ordinario en obstetricia.

Beecher y colaboradores basados en observaciones clínicas muy cuidadosas sostienen que en pacientes jóvenes se puede hacer una apreciación aproximada del volumen de sangre perdida estableciendo una "relación entre los signos clínicos y la magnitud del déficit de volumen en el shock hemorrágico" (4). Y así establece la clasificación de la severidad o gravedad del shock en Ninguna, Poco, Moderada y Mucha, (como lo veremos en el Cuadro 1), en la cual los cambios volumétricos anotados se basan en las observaciones

* Trabajo presentado al IX Congreso de Anestesia, en Medellín, el 16 de Agosto de 1969.

** Médico Jefe del Departamento de Obstetricia y Ginecología del Hospital San Juan de Dios, de Cúcuta.

*** Médico Jefe del Departamento de Anestesia de la Clínica de Maternidad de Medellín "Luz Castro de Gutiérrez". 


\section{CUADRO N: 1}

\section{RELACION ENTRE LOS SIGNOS CLINICOS Y LA MAGNITUD DEL DEFICIT DE VOLUMEN} EN EL SHOCK HEMORRAGICO

\begin{tabular}{|c|c|c|}
\hline $\begin{array}{l}\text { Gravedad } \\
\text { del shock }\end{array}$ & Hallazgos clínicos & $\begin{array}{l}\text { Disminución porcentual } \\
\text { del volumen sanguíneo }\end{array}$ \\
\hline NINGUNA & $\begin{array}{l}\text { Ninguno. } \\
\text { Pérdida normal de sangre. }\end{array}$ & $\begin{array}{l}\text { Más del } 10 \% \\
(500 \text { c.c.) }\end{array}$ \\
\hline POCA & $\begin{array}{l}\text { Taquicardia mínima. } \\
\text { Ligera disminución de la presión arterial. } \\
\text { Ligera vasoconstricción periférica con pies y manos frías. }\end{array}$ & $\begin{array}{l}\text { Del } 15 \text { al } 25 \% \\
(750 \text { a } 1.250 \text { c.c. })\end{array}$ \\
\hline MODERADA & $\begin{array}{l}\text { Taquicardia ( } 100 \text { a } 120 \text { pulsaciones por minuto). } \\
\text { Disminución de la presión del pulso. } \\
\text { Presión arterial sistólica de } 90 \text { a } 100 \mathrm{~mm} \text {. de } \mathrm{Hg} \text {. } \\
\text { Inquietud. } \\
\text { Diaforesis intensa. } \\
\text { Palidez. } \\
\text { Oliguria. }\end{array}$ & $\begin{array}{l}\text { Del } 25 \text { al } 35 \% \\
(1.200 \text { a } 1.750 \text { c.c. })\end{array}$ \\
\hline MUCHA & $\begin{array}{l}\text { Taquicardia (más de } 120 \text { pulsaciones por minuto). } \\
\text { Presión arterial sistólica menor de } 60 \mathrm{mmg} \mathrm{Hg} \text {. y general- } \\
\text { mente imposible de obtener con manguito de esfigmoma- } \\
\text { nómetro. } \\
\text { Estupor. } \\
\text { Palidez extrema. } \\
\text { Extremidades frías } \\
\text { Anuria. }\end{array}$ & $\begin{array}{l}\text { Mayor del } 50 \% \\
(2.500 \text { c.c. })\end{array}$ \\
\hline
\end{tabular}

clíincas, y la pérdida de volumen sanguíneo de 7 por ciento en un varón de 70 kilos de peso y de complexión mediana.

Es evidente que en el shock hemorrágico se debe reemplazar la sangre perdida y elevar así el volumen sanguíneo efectivo (5), si bien la indicación indiscutible del uso de sangre es la necesidad de mejorar la capacidad de transporte de Oxígeno (6) (7) (8). Pero encontramos una serie de factores que dificultan este tratamiento como son la falta de sangre o la existencia insuficiente, la dificultad y la demora en la clasificación a más de los peligros inherentes a la transfusión.

Conocedores de la eficacia del empleo de los expansores del plasma para el tratamiento del preshock y para la iniciación del tratamiento del shock establecido, decidimos usar un expansor del plasma (el Haemaccel) en estas pacientes, teniendo siempre en mente que cuando suministramos líquidos para el tratamiento del shock hipovolémico éstos deberán asegurar fundamentalmente la suficiente oxigenación de los tejidos y por lo tanto deberán reunir dos características principales: a) ser capaces de combatir la hipovolemia eficazmente y evitar así que con la disminución del volumen circulante y del retorno venoso el gasto cardíaco baje a niveles críticos lo que traería como consecuencia el desencadenamiento de la serie de eventos que conducen al establecimiento del estado de shock; y b) no interferir el mecanismo de transporte de oxígeno. 
No olvidemos que el tratamiento del shock se debe instaurar, de ser posible, apenas se presenten los síntomas premonitores concomitantes 0 consecutivos a la hemorragia conocidos con el nombre de amenaza de shock o preshock (taquicardia con presión sanguínea aún normal).

\section{Expansores de plasma}

Para que un expansor de plasma se pueda usar como substituto debe tener propiedades muy similares a las del plasma: no debe abandonar el torrente circulatorio con demasiada rapidez, pero una vez estabilizada la circulación, debe ser eliminado sin demora; debe tener una presión coloide osmótica definida y sus propiedades físicas generales deben hacerlo apto para la infusión; excepto por sus propiedades físicas debe ser morfológicamente inerte; no debe tener efecto antigénico, alergénico ni pirógeno; no debe interferir los grupos sanguíneos ni la aglutinación cruzada sanguínea; se podrá almacenar por largo tiempo y deberá tolerar variaciones en la temperatura sin perder efectividad; será fácilmente esterilizable. Y sólamente una substancia macromolecular puede responder a estos requerimientos.

El volumen plasmático no puede específicamente ser aumentado a menos que el fluido administrado contenga un agente coloide. La administración de solución salina a un sujeto que ha perdido sangre, reexpandería el volumen del fluído extracelular, pero virtualmente toda la expansión será confinada al compartimiento intersticial donde la pérdida no ha ocurrido en primera instancia (9).

El Haemaccel, con un buen número de requisitos a su favor que le hacen un buen expansor plasmático, disminuye la viscosidad de la sangre, mejora la microcirculación como también la perfusión renal y por consiguiente la eliminación urinaria, y evita el fenómeno de Sludge.

El Haemaccel es una asociación de polipéptidos a partir de gelatina altamente purificada, ligados con un di-isocianato; su molécula es más o menos esférica, de peso molecular aproximado a 35.000; se mantiene fluído a temperaturas hasta de 3 grados centígrados; es totalmente exento de antigenicidad; su permanencia en el torrente circulatorio, mediante estimaciones de hidroxiprolina es de 4 a 5 horas en promedio $(10,11,12$, $13,14,15)$.

\section{Material y Métodos}

Hemos tratado 50 pacientes: 15 de los Servicios de Maternidad y de Ginecología del Hospital San Juan de Dios de Cúcuta, y 35 de la Clínica de Maternidad de Medellín "Luz Castro de Gutiérrez", de Medellín.

En 34 la tensión arterial máxima estaba entre 4 y 7 y la mínima entre 2 y 5 . Y en las 16 restantes la tensión arterial máxima oscilaba entre 0 y 3.

Siguiendo las experiencias de Beecher y colaboradores, a 17 pacientes con una pérdida sanguínea aproximada entre 15 y $25 \%$ administramos únicamente Haemaccel; a 8 con pérdidas aproximadas del 25 al 35\% aplicamos inicialmente Haemaccel y luego solución de Hartmann o Dextrosas; y a 25 con pérdidas aproximadas superiores al $35 \%$ les aplicamos inicialmente Haemaccel seguido de sangre total.

A doce pacientes administramos entre 500 y $1.000 \mathrm{cc}$. de Haemaccel. A 34 administramos entre 1.000 y 2.000 cc. y a cuatro les debimos infundir entre 2.500 y $4.000 \mathrm{cc}$. 
A 12 pacientes les hicimos cateterimso de las venas del pliegue del codo con miras a controlar la presión venosa central.

Tuvimos necesidad de digitalizar a dos pacientes, y a dos les administramos ácido amino caproico.

De acuerdo a las normas de Weil (16) y de su célebre aforismo VIP (Very Important Pause o Ventilation Infusion Pomp = Ventilación Infusión Bomba), colocamos a todas nuestras pacientes un catéter vesical, catéter para presión venosa central a doce, posición horizontal con ligera elevación de las piernas (de 10 a 20 grados) más oxigenación. Les administramos inmediatamente Haemaccel a presión para infusión rápida. A ninguna de las pacientes aplicamos vasopresores.

El tratamiento lo instituímos con la mayor rapidez posible apenas observamos los primeros signos del preshock o inmediatamente ingresaba la paciente al Servicio en preshock o en shock establecido.

En todos los casos administramos los primeros $500 \mathrm{cc}$. a presión con la ayuda del brazalete del tensiómetro y luego seguimos administrándolo a razón de 60 a 80 gotas por minuto. La administración de soluciones electrolíticas o Dextrosadas o la sangre total la practicamos con cierta rapidez o aún a presión, según la urgencia o gravedad de cada paciente.

\section{Resultados}

Tanto en las 17 pacientes a quienes administramos únicamente Haemaccel como a las 8 a quienes dimos Haemaccel más Hartmann o Dextrosa, obtuvimos rápidamente cifras tensionales entre 8 y 10 al terminar su aplicación. Resultados similares obtuvimos en las 25 pacientes a quienes administramos Haemaccel sangre total, si bien en seis de ellas se presentaron luego fuertes caídas tensionales que nos obligaron a suministrar repetidas dosis de Haemaccel a presión y aún sangre a presión: una de estas pacientes no respondió después de esta caída tensional, hizo paro cardíaco y falleció.

La presión venosa central en las doce pacientes a quienes la practicamos, era inicialmente de 3 a 5; subió hasta 8 y $10 \mathrm{~m} . \mathrm{m}$. con la sola infusión de Haemaccel.

Quiere éllo decir que en nuestra casuística obtuvimos una franca y sostenida respuesta a la aplicación de Haemaccel en infusión rápida y suficiente, a presión, en el $98 \%$ de los casos.

En ninguna de nuestras pacientes observamos efectos pirógenos ni alérgicos.

Tenemos pruebas fehacientes para no atribuir al Haemaccel las alteraciones de los mecanismos de coagulación que encontramos en dos de nuestras pacientes.

\section{Comentarios}

En pacientes con colapso vascular grave consecutivo a pérdida aguda de un volumen apreciable de sangre, la rapidez con que se instale el tratamiento es de un valor inapreciable.

Y esta iniciación inmediata del tratamiento a base de transfusión sanguínea no la podemos obtener en nuestros medios hospitalarios. Además, bien conocemos los peligros de las sangres de Banco: en oportunidades hay falta de personal bien entrenado que no nos da seguridad sobre omisión de error humano; el pH bajo de 6 a 6,5 y el aumento de potasio que pueden producir o aumentar 
una acidosis metabólica; o ser un riesgo depresivo miocárdico por el peligro de la hipotermia selectiva del miocardio que se produce cuando se transfunden rápidamente grandes cantidades de sangre de banco y que por la urgencia no es posible calentarla; el peligro de las hepatitis; la posible presencia de criobacterias, de plasmodium o de otros hemoparásitos; la pérdida de factores de coagulación, son todas posibilidades muy significativas e inquietantes que nos hacen pensar y recapacitar muy seriamente sobre el uso de transfusiones de sangre solamente bajo indicaciones estrictas y de acuerdo a las condiciones y características de cada paciente.

Todas estas consideraciones hacen que empecemos el tratamiento del shock hemorrágico con aplicación de expansores de plasma, tratamiento que podemos iniciar a los pocos segundos de haber observado los primeros signos de alarma.

La rapidez en la administración del expansor de plasma que hemos usado (Haemaccel) la hemos logrado con facilidad ya que su envase plástico nos permite enrollarle en su rededor el brazalete de un tensiómetro para darle así presión y velocidad a la infusión.

El plasma liofilizado, por su alto costo, no está al alcance de nuestras instituciones; tampoco tenemos existencia de plasma fresco.

La albúmina a más de ser de elevado costo, tampoco la tenemos en nuestro medio.

No olvidemos que las transfusiones tanto de volumen reducido como mativas o a presión las debemos emplear únicamente cuando estén estrictamente indicadas.

\section{Conclusiones}

Basados en nuestras observaciones $y$ en estudios muy serios ya bien conocidos y divulgados tanto en publicaciones científicas como en comunicaciones a reuniones internacionales podemos concluir:

El Haemaccel, expansor de plasma polimerizado, es un elemento de gran valor en la fase inicial del tratamiento del shock hemorrágico por la rapidez en la instalación y en la administración; porque posee todos los requisitos que debe llenar un expansor de plasma para ser ideal; por su fácil manejo en cualquier sitio donde nos encontremos y por la eficacia que pudimos comprobar en las pacientes estudiadas.

Es pues una forma de ataque rápido, eficaz y seguro en la etapa inicial del shock hemorrágico, de una simplicidad tal que puede bien decirse que no hay lugar donde no se pueda utilizar.

\section{Resumen}

Se hacen algunas consideraciones sobre el shock hemorrágico y su tratamiento.

Se estudian las propiedades de una gelatina polimerizada, el Haemaccel, y su empleo en la fase inicial del tratamiento del shock hemorrágico en gineco obstetricia.

Se analizan los resultados obtenidos con este tratamiento en cincuenta pacientes con pérdida sanguínea aproximada del 10 al 35\%.

Se hacen comentarios.

Se dan conclusiones.

\section{BIBLIOGRAFIA}

1 RATNOFF, O. D., PRITCHARD, J. A., and COLOPY, E. J. Hemorragic states during 
pregnancy. New England J. Med. 253: 63, 1955.

2 RODRIGUEZ DE LA FUENTE, F. Choque en Obstetricia. Ginec. Obstet. Méx. 163: 179, 1968.

3 BEECKER, H. K., SIMEONE, F. A., BUERNETT, C. H., SHAPIRO, S. L., SULLIVAN, E. R. and MALLORY, T. B. The internal State of the severely wounded man on entry to the most forward Hospital. Surgery. 22 : 672,1947 .

4 BEECHER y col. Tomado del libro Diagnosis and Treatment of Shock, by Harry Weil and Herbert Shubin. Ed. The Williams and Wilkin Co., 1967.

5 MASON EDWARD E. Insuficiencia circulatoria (Shock). En el libro Metabolismo del agua y los electrolitos en clínica, de John H. Bland. Ed. Interamericanas S. A. México. Pág. 497, 1965.

6 ESPINOSA DE LOS REYES, V., RODRIGUEZ ARGUELLES, J., DELGADO URDAPILLETA, J. y RAMIREZ SOTO, E. Manejo de la enferma con shock en Obstetricia. Ginec. Obstet. Méx. 146: 659, 1968.

7 ANGULO RODRIGUEZ, L. Apreciaciones sobre el shock en obstetricia. Rev. Col. Obst. Gin. 6: 199, 1954.

8 VELASCO CHIRIBOGA, A. Shock. Rev. Col. Obst. Gin. 17: 223, 1966.

9 GOODMAN, L. y GILMAN A. Bases farmacológicas de la terapéutica. Unión Tipográfica Editorial Hispano Americana. 3a. Ed. Pág. 769.
10 ANDREUCCI, S. Tipizzazione del sangue e I'uso di un nuovo plasmaexpander: I'Emagel. Minerva Anestesiologica. 29: 342, 1963.

11 ROGERS, C. J., KIMMEL, J. R., HUTSCHIN, M. E. and HARPER, H. A. A Hydroxyproline method of analysis for a modified gelatin in plasma and urine. J. Biol. Chem. 206: $553,1954$.

12 PANNACCIULLI, E. e SERRA, G. C. Azione sul circolo di un nuovo sostituto plasmatico constituito de polimerizzati di gelatina scissa (Emagel). Minerva Anestesiologica. 29: 349, 1963.

13 DE CECCO, L. e LUCISANO, F. Possibilitá di impiego di nu nuovo sostituto plasmatico in ostetricia e ginecologia. Minerva Anestesiologica. 29: 421, 1963.

14 MERTZ, D. P. Estudios químicos y experimentales sobre el efecto de un nuevo expansor de plasma en la hemodinámica renal y los mecanismos de concentración de la orina. Arzneimittelforschung (Drug Res) 12 : 489, 1962.

15 EBERLEIN, J. y DOBBERSTEIN, H. Estudios de la circulación en donantes de sangre, después de la infusión rápida de un nuevo expansor de plasma. Arzneimittelforschung 12: 494, 1962.

16 WEIL, H. Conferencia dictada el sábado 29 de abril de 1969 en el Simposio Internacional sobre shock y uso de substitutos de plasma. Ciudad de México, L. F. 